\title{
Der finnische Dativgenitiv auf der Basis des wolgafinnischen Genitivs
}

Nobufumi Inaba: Suomen datiivigenetiivin juuret vertailevan menetelmän valossa. [Der finnische Dativgenitiv im Licht der vergleichenden Methode.] Suomalais-Ugrilaisen Seuran Toimituksia 272. Suomalais-Ugrilainen Seura. Helsinki 2015. 413 S. +62 S. Anhang (Diss.)

Die vorliegende Untersuchung ist eine zusammenfassende Fortsetzung der 1997 erschienenen und hauptsächlich auf den finnischen Dativgenitiv konzentrierten Arbeit von Nobufumi Inaba. Über die Dativität des Dativgenitivs wurde in Finnland erstmals 1649 geschrieben; die Wurzeln des Genitivs wurden seit 1880 zeitweise sehr intensiv untersucht, und die Entstehung des Genitivs wurde je nach der Schule auf die finnischugrische Ursprache oder auf das Ururalische datiert. Deshalb ist die vorliegende Untersuchung äußerst interessant; dies beginnt bereits mit der Frage, wie es dem Verfasser gelingt, das Feld so einzugrenzen, dass er nicht von den konkurrierenden, aber bisher fruchtlosen Hypothesen gefangen genommen wird.
Das 1. Kapitel oder die Einleitung der Untersuchung von Nobufumi Inaba beginnt denn auch mit einer Darstellung des Forschungshintergrunds. Dort heißt es, dass die sog. finnisch-wolgaischen Sprachen, also Ostseefinnisch, Saamisch, Mordwinisch und Mari, im Hinblick auf die Mittel zum Ausdruck der Besitzverhältnisse eine Gesamtheit bilden, da gerade in diesen finnisch-ugrischen Sprachen in der adnominalen Besitzkonstruktion der Besitzer mit dem auf $n$ zurückgehenden Genitiv (kurz N-Genitiv) markiert wird, z. B. finn. pojan kirja, das Buch des Jungen. Als unterscheidende/verbindende Merkmale innerhalb dieser Gesamtheit fungieren fünf mit Possessivsätzen verbundene Eigenschaften, deren Funktionsbereiche die Abb. 1 (S. 16) veranschaulicht. Die Eigenschaften und ihre Funktionen sind die folgenden: a) der Lokalkasus als Markierer des Besitzers im Possessivsatz (z. B. finnisch Pojalla on kirja, der Junge hat ein Buch') verbindet alle saamischen und alle ostseefinnischen Sprachen mit Ausnahme des Südsaamischen und des Livischen; b) der N-Genitiv als Markierer des Besitzers im Possessivsatz verbindet Südsaamisch, 
Mordwinisch und Mari; c) der Dativ markiert den Besitzer im Possessivsatz im Livischen; d) eine Eigenschaft, die Mordwinisch und Mari verbindet und von den anderen westlichen finnisch-ugrischen Sprachen unterscheidet, ist das an den Besitz angefügte Possessivsuffix; e) das Süd-, Ume-, Pite- und Lulesaamische verbindet die Möglichkeit, alternativ einen auf dem ,besitzen'Verb aufbauenden Possessivsatz zu verwenden.

So wird für die Untersuchung ein formales und zugleich gut strukturiertes und zuverlässiges Grundschema geschaffen, das die Untersuchung im Prinzip von der Bürde der konkurrierenden Hypothesen über die Entstehung des $N$ Genitivs befreit. Die Logik dieses auf realen Eigenschaften basierenden Grundschemas hebt den Dativgenitiv des Finnischen in keiner Weise hervor; die Entstehung des Dativigenitivs wird so automatisch vor allem der Entwicklung des Finnischen zugeordnet. Daraus, dass der $N$-Genitiv als Grundbegriff der Untersuchung hervorragend funktioniert, folgt, dass zur Zeit der Entstehung des finnischen Dativgenitivs der $\mathrm{N}$-Genitiv der finnisch-wolgaischen Sprachen bereits entstanden war und eine Schlussfolgerung, die seinen Ursprung eingehender beleuchtet, zumindest auf dieser Basis nicht zu erreichen ist.
Dies ist das erste wichtige Ergebnis von Inabas Untersuchung, das jedoch nicht deutlich sichtbar wird, weil das Fehlen des $N$ Genitivs in den permischen und ugrischen Sprachen und sein Vorhandensein in den samojedischen Sprachen, die aus dem Bild und auch aus dem Grundschema ausgeschlossen werden, vier konkurrierende Erklärungsmuster zur Entstehung des $\mathrm{N}$-Genitivs verursacht haben; Inaba kann nicht umhin, sie zu behandeln, denn sie sind ja in gewisser Weise ein wichtiger Teil der Forschungsgeschichte. Er stellt jedoch bereits in der Einleitung fest, dass

hinsichtlich der Forschung der größte Mangel bisher jedoch war, dass man es nie unternommen hat, zu klären, ob der finnische Dativgenitiv tatsächlich alter Abstammung ist. Diese Annahme wurde nie in Frage gestellt, obwohl in den verwandten Sprachen keine zuverlässigen Spuren des Dativgenitivs gefunden und die Möglichkeit einer internen Entwicklung im Finnischen oder des Einflusses fremder Sprachen nicht berücksichtigt wurden (S. 18).

Als Untersuchungsziel leitet sich daraus ab,

eine objektive Darstellung und Analyse des finnischen Dativgenitivs sowie der mit seiner Herkunft verbundenen Umstände vorzulegen und aufgrund der Analyse eine Schlussfolgerung zu 
ziehen, die das Rätsel des Ursprungs des $N$-Genitivs der finnisch-wolgaischen Sprachen im Hinblick auf den Dativgenitiv beleuchten (S. 19).

Inaba nennt die folgenden Forschungsgegenstände: 1) der finnische Dativgenitiv und seine Stellung bei der Kasusmarkierung des Objekts, 2) die Verwendung des Dativs im Livischen als eines der Ausdrucksmittel für Besitzverhältnisse, 3) die Ausdrucksmittel des Südsaamischen, 4) die Ausdrucksmittel für Besitzverhältnisse im Mordwinischen und Mari sowie zusätzlich 5) die Kasusmarkierung des direkten und indirekten $\mathrm{Ob}$ jekts im Altschwedischen und im älteren Neuschwedischen; diese werden entsprechend in den Kapiteln 3-7 behandelt. Die Kasussyntax dieser Forschungsgegenstände wird vergleichend untersucht. Die Korpora zum Dativgenitiv der alten finnischen Schriftsprache, zum Dativ des Livischen und zum Genitiv des Südsaamischen werden, soweit sie zugänglich waren, vollständig ausgewertet, für Mordwinisch, Mari und Schwedisch wird stichprobenartiges Material verwendet. Zur Bestätigung der Hintergrundinformationen werden ferner ter-, kildin-, skolt-, lule- und umesaamische Texte sowie wotisches und ingrisches Material herangezogen.
Die Untersuchung erweitert sich also vom engen fennistischen, hauptsächlich auf die alte finnische Schriftsprache beschränkten Feld auf das Gebiet der finnischugrischen Sprachforschung und erstreckt sich darüber hinaus noch auf den Bereich der skandinavischen Sprachen, vornehmlich der alten schwedischen Schriftsprache.

Die Einleitung enthält ferner kurze Charakterisierungen der Methode (die sog. einfache Kasussyntax und die darauf beruhende vergleichende Methode), des Untersuchungsmaterials (allein das Quellenverzeichnis am Ende des Bandes umfasst 20 Seiten), des Aufbaus der Untersuchung sowie der Besitzverhältnisse und ihrer Ausdrucksmittel. Aus der Sicht des Lesers ist die Darstellung der Besitzverhältnisse und ihrer Ausdrucksmittel aufschlussreich und auch wichtig, da in der Untersuchung die Begriffe verschiedener Theorien einander begegnen.

Das Kapitel 2 über das Rätsel des Ursprungs des $N$-Genitivs und über seinen Hintergrund bietet einen äußerst detaillierten Überblick über die Entwicklung der vier Erklärungen für die Entstehung des Genitivs. Es handelt sich um die folgenden: (1) Genitiv und Instruktiv sind ursprünglich ein und derselbe Kasus, (2) der Genitiv geht auf das frühere Possessivadjektiv zurück, (3) der 
Genitiv (und der Instruktiv) ist aus dem alten ${ }^{*}-n A$-Lokativ entstanden, (4) der Genitiv ist aus dem Lativ entstanden. Die Reihenfolge der Erklärungen orientiert sich nicht an ihrem Alter, sondern eher an ihrer Popularität in steigender Folge. So ist die erste die jüngste und 1928 entstanden: K. B. Wiklund hielt es für möglich, dass Instruktiv und Genitiv aufgrund der gemeinsamen temporalen, lokalen, modalen und possessiven Funktionen derselbe Kasus sind; später wurde versucht, auch den Lativ hinzuzufügen. Die zweite Erklärung legte 1865 F. J. Wiedemann vor, der bemerkte, dass im Mordwinischen gewisse Wörter mit dem Suffix ń-, wenn sie attributiv oder vor einer Postposition verwendet werden, sowohl die Genitivform des Substantivs als auch ein Adjektiv darstellen können, und da das Suffix $n$ - ein Adverb zu einem Adjektiv machen kann, interpretierte er es als ursprüngliches Ableitungssuffix zur Bildung von Adjektiven. Die dritte Erklärung legte 1873 Michael Weske vor, der verschiedene $n$-Elemente enthaltende Suffixe auf den alten -na/-nä-Lokativ zurückführte. Übrigens ist $\mathrm{zu}$ beachten, dass eine Besonderheit seiner Methode die morphemweise Interpretation der Wortformen ist. Die vierte, sog. Lativhypothese stellte Erkki Almberg 1871 auf, der aufgrund des Wechsels von Dativgenitiv und Al- lativ im Finnischen den Genitiv auf den Lativ zurückführte. Alle vier Erklärungen haben Anhänger gefunden und wurden weiterentwickelt, und neue Ideen wurden auch von Anhängern konkurrierender Erklärungen in Gebrauch genommen. Im Allgemeinen enthalten die Erweiterungen der vier Erklärungen sowohl gemeinsame als auch gegensätzliche Elemente. Inaba fasst die Eigenschaften und Schwächen der bisherigen Hypothesen in einer Tabelle zusammen, in der die Schwächen der Lativhypothese jedoch nicht sichtbar werden.

Kapitel 3 befasst sich mit dem finnischen Dativgenitiv. Dieser wird äußerst sorgfältig dargestellt, zunächst anhand der Quellentexte, also zusammenfassend durch die Zeiten: Belege aus dem Zeitraum 1500-1650, aus der Zeit nach 1650, und gleichzeitig werden die Beispiele gegliedert in solche, die in der Position des Wohinkasus, also des finnischen Allativs, und solche, die in der Position des Lokalkasus, also des finnischen Adessivs auftreten, wobei die in der Stellung des Allativs auftretenden noch in drei Unterkategorien gegliedert werden. Das ist keine leichte Aufgabe, ich bezweifle, dass selbst finnische Muttersprachler fähig sind, alle mit dem Verb olla , sein' auftretenden Genitivformen eindeutig als Ausdruck des Woher- oder des 
Lokalkasus einzuordnen, wie z.B. bei dem Beleg eli mitä meidän sijtä hywä on / että me händä rucoilem?, der auf S. 51 eine eigene Nummer (vi) vielleicht eben deshalb erhält, weil er sich gewissermaßen von dem Beleg (v) Kijtos olcon Jumalan ..., unterscheidet, wo der Genitiv eindeutig den Wohinkasus vertritt. Aufgrund meiner eigenen Muttersprache akzeptiere ich in diesem Fall unter bestimmten Voraussetzungen beide Möglichkeiten. Glücklicherweise sind solche Fälle nicht sehr häufig.

Dieses Kapitel ist schlichtweg verblüffend, wir erhalten ein Bild vom Vorkommen des Dativgenitivs mit jedem Verb, das in den im 16.-17. Jahrhundert herausgegebenen Werken begegnet, und im Anhang 1 sind zudem alle Einzelfälle aufgeführt, in denen es sich nicht um das Subjekt eines Infinitivs handelt. Die Belege für den Dativigenitiv werden in sieben Belegtypen gegliedert. Im Korpus finden sich auch Belege für das gleichzeitige Auftreten des Genitivs mit dem Allativ oder Adessiv; Inaba charakterisiert dieses Phänomen als funktionale Kongruenz zweier Kasus. Obwohl die Verwendung des Dativgenitivs im 16.-17. Jahrhundert tendenziell zurückgeht, finden sich Belege auch aus späterer Zeit, auch in den Werken von Autoren, die im 19. Jahrhundert geboren wurden. In der zusammenfassenden Darstellung des in Agricolas Werken vorherrschenden Systems zum Ausdruck von Besitzverhältnissen wird auch die Verwendung des Elativs neben dem Dativgenitiv als habitive Kasusform vorgestellt, und auch sonst werden in dem $\mathrm{Ka}$ pitel vielerlei Sachverhalte und Probleme beleuchtet oder gelöst. Aus sprachgeschichtlicher Perspektive darf als wichtigstes Ergebnis dieses Kapitels gelten, dass seit Agricola als habitiv-possessiver Wohinkasus neben dem Genitiv der Allativ, als Lokalkasus neben dem Genitiv der Adessiv und als Trennungskasus neben dem Elativ der Ablativ verwendet wurde, also dieselben Kasus wie im heutigen Finnisch.

Mich irritiert jedoch der im letzten Teil des Kapitels auf S. 96 vorgebrachte Gedanke

Sofern das hohe Alter des Dativgenitivs, welches die in Kapitel 2.4. dargestellte LAT-Hypothese postuliert, zutrifft, handelt es sich um eine systematische überschneidende Verwendung des Objektkasus, die besteht, seit der $m$-Akkusativ zum $n$-Akkusativ wurde. Der Dativgenitiv hat seine Ausdruckskraft auch dann nicht verloren, nachdem der konkurrierende habitive Wohinkasus, der Allativ, Teil des Sprachsystems geworden war.

Mit der LAT-Hypothese wurde in der Regel versucht, die Entstehung des Genitivs zu erklären; den Fen- 
nisten der letzten Jahrzehnte zufolge ist das hohe Alter, d. h. die Entstehung des Dativgenitivs ein jüngeres Phänomen als die Verschmelzung des $m$-Akkusativs mit dem Genitiv und auch jünger als die Entstehung des Dativs im Livischen.

Kapitel 4 über den Dativ im Livischen beginnt mit einer Darstellung des Hintergrunds, bei dem es sich um die Theorien über die Entstehung des kurlandlivischen Dativs: (1) die Entstehung des Dativs aus dem Genitiv, (2) aus dem Lokativ, (3) aus dem Dativ des Lettischen, (4) aus dem Terminativ des Estnischen, (5) aus der Verschmelzung von Genitiv und Lokativ. Inaba ist der Ansicht, dass wegen Mangel an Informationen keine dieser Theorien akzeptiert werden kann. Er hat recht, die Frage ist eher, wie sich die Liste reduzieren lässt. Zugleich hat niemand die sprachwissenschaftliche Literatur zum livischen Dativ sowie die gesamte gedruckt vorliegende, authentische livischsprachige Literatur so sorgfältig durchgesehen wie Inaba. Da ein großer Teil des verwendeten Materials in phonetischer Transkription vorliegt, war die Vereinfachung der Schreibweise zweifellos äußerst mühsam.

Inaba legt die bisher beste $\mathrm{Ge}$ samtdarstellung der Morphologie des Dativs im Kurlandlivischen und Salislivischen vor, wobei er unter anderem besonderes Augen- merk auf die kurzen Dativformen legt und eine Reihe genauer und bemerkenswerter Beobachtungen bietet. Er liefert übrigens auch eine interessante Erklärung dafür, weshalb Lauri Kettunen im grammatischen Teil seines Wörterbuchs die kurzen Dativformen der livischen Personalpronomina nicht angegeben hat. Inaba hat die Belege für den Dativ sorgfältig gruppiert und richtet dabei das Hauptaugenmerk auf das habitiv-possessive Adverbial und Prädikativ (dafür finden sich in seinem Material 9663), auf nezessive, permissive und gerundiale Konstruktionen, andere Subjekte der Nominalformen des Verbs und Prädikative der Nominalformen. Inaba legt eine dankenswerte Ergänzung zu meiner Typisierung der Nezessivkonstruktionen vor und zugleich eine bessere Neuordnung ihrer Kennzeichen. Inaba hat hier übrigens den in Kettunens Wörterbuch verzeichneten Infinitiv pi'ddõ wieder in Gebrauch genommen, um die livischen Entsprechungen des finnischen Nezessivverbs pitää, nämlich die Stammformen pid̄im und pidìiks, die zwei Modi vertreten, zu vereinen. Es gibt im Livischen jedoch keinen Beleg für die Verwendung einer solchen gemeinsamen Form. Es ist mir nicht gelungen, auch nur einen Liven zur Produktion dieses Infinitivs zu bewegen, dessen Existenz zudem 
auch von Pētõr Damberg bestritten wurde. Daher habe ich den Verdacht, dass es sich um ein Ergebnis der theoretischen Überlegungen Kettunens handelt, wahrscheinlich brauchte er das Lemma.

Inaba stellt aufgrund der Beschreibung und Analyse des livischen Dativs fest, dass der livische Dativ und der finnische Genitiv mehr gemeinsame Verwendungsgebiete haben als bisher angenommen. Ich stimme dem zu. Ich stimme Inaba auch zu, wenn er auf S. 169 schreibt,

der livische Dativ hat zwar sowohl die Funktion des finnischen Genitivs als auch die des Essivs, doch es ist naheliegender, dies als Resultat des $\mathrm{Zu}$ sammenfalls von zwei Kasusformen zu betrachten.

Als Betriebsunfall betrachte ich dabei die Begründung:

weil eine der Verwendung des Essivs entsprechende Verwendung des Dativs, im Gegensatz zu Kettunens Behauptung, ausschließlich bei temporalen Adverbialen zu beobachten ist.

Da sich richtigerweise mit dem finnischen Essiv übersetzte nichttemporale Dativformen auch unter Inabas Belegen finden, z. B. (206), (207), (216), (224-238), handelt es sich wahrscheinlich um eine veraltete Passage, die versehentlich nicht gestrichen wurde.
Kapitel 5 befasst sich mit den Possessivsätzen im Süd- und Umesaamischen, von denen es drei Typen gibt, sowie vor allem mit der Verwendung des $n$-Genitivs in den südsaamischen Possessivsätzen und mit dem Verhältnis dieser zu anderen Possessivsätzen, in denen der Besitzer mit der Genitivendung markiert wird. Auf Seite 177 findet sich die zweite Abbildung 1 des Buches über die Verteilung der saamischen Sprachen nach den Possessivsatztypen und dem habitiven Kasussystem. Die Analyse der Verwendung genitivischer Possessivsätze im Südsaamischen ergibt, dass die Verwendung eines solchen Satzes bei unbelebtem Besitz den südsaamischen Genitiv vom Dativgenitiv des Finnischen unterscheidet, ihn aber mit dem Genitiv im Mordwinischen und Mari verbindet.

Im Kapitel 6 werden die Besonderheiten der Possessivsätze im Mordwinischen und Mari vorgestellt - in beiden Sprachen kann die Person des Besitzers auch allein mit einem Possessivsuffix angegeben werden, das im Mordwinischen auch die Person des Besitzes ausdrückt. Der Vergleich der Ausdrucksmittel für Besitz- und Innehabungsverhältnisse im Mordwinischen und im Mari mit den entsprechenden Ausdrucksmitteln im Südsaamischen, Finnischen und Livischen zeigt, dass der Genitiv 
des Mordwinischen und des Mari vom finnischen Dativgenitiv weit entfernt ist. Die Lativendung $-\eta$ und die Genitivendung - $n$ in den nordwestlichen Dialekten des Mordwinischen gehen nicht auf dieselbe Endung zurück und können nicht mit dem finnischen Dativgenitiv verbunden werden.

Kapitel 7 behandelt die Große Katastrophe des schwedischen Kasussystems, d.h. mit den Veränderungen, die zur Vereinfachung des alten, aus vier Kasusformen bestehenden Systems und zur Vermischung verschiedener Kasusformen führten, vor dem Hintergrund des Isländischen, des mittelalterlichen Norwegischen und des Deutschen. Da die Kapitel 4-6 die Frage nach dem Ursprung des finnischen Dativgenitivs nicht klären und da die Entstehung der alten finnischen Schriftsprache gerade in die letzte Zeit des Nebeneinanders der alten und der neuen Sprachform des Schwedischen fällt, nimmt Inaba an, dass unter dem Einfluss des Schwedischen die Praxis entstand, das Objekt und den Empfänger mit derselben Kasusform zu kennzeichnen, und dass gerade der Genitiv des Objekts von Personalpronomina und von Substantiven im Singular die Grundlage abgab. Die dativische Verwendung des Genitivs Plural der Substantive erklärt sich aus der Analogie zum Singular und $\mathrm{zu}$ den Personalpronomina. Als Beweis für Inabas Hypothese der Entstehung des Dativgenitivs eignen sich die in Kapitel 3 dargestellten sog. Merkwürdigkeiten, in denen sich die Kennzeichnung des indirekten Objekts im Schwedischen widerspiegelt.

Kapitel 8 analysiert zunächst anhand der Erfahrungen und Ergebnisse der Untersuchung sachgemäß die Hauptlinien und Schwächen der früheren Forschung. Aufgrund seiner Untersuchung gibt Inaba einen vergleichenden Überblick über die gemeinsamen und besonderen Züge des finnischen Dativgenitivs, der an Besitzsätzen und -konstruktionen beteiligten Kasusformen und Verben sowie Beschränkungen im Livischen, Südsaamischen, Mordwinischen und Mari. Die genitivischen Besitzsätze im Saamischen, Mordwinischen und Mari weisen Gemeinsamkeiten auf, die sie von den ostseefinnischen Sprachen unterscheiden. Daraus ergibt sich der Schluss, dass das Resultat der vergleichenden Methode eindeutig ist: In den verwandten Sprachen, in denen es einen $\mathrm{N}$-Genitiv gibt, finden sich keine Spuren des Dativgenitivs. Es folgt eine detaillierte, logische Darstellung darüber, wie man begann, im Finnischen den als Kasus des (direkten) Objekts verwendeten Genitivs nach dem neuen Muster des Schwedischen auch als habiti- 


\section{Tiit-Rein Viitso}

ven Woherkasus oder Dativgenitiv zu verwenden. Der finnische Dativgenitiv ist also eine unter dem Einfluss des Schwedischen entstandene Innovation. Inaba erklärt, das Gewicht seiner Hypothese, also seiner Erklärung der Entstehung des Dativgenitivs sei

sehr gering, sie widerlegt nur die Hypothese vom lativischen Ausgangspunkt des Genitivs, zumindest im Hinblick auf den finnischen Dativgenitiv.

Dabei ist gerade die Erkenntnis, dass der finnische Dativgenitiv kein altes finnisch-ugrisches Erbe ist, sondern hauptsächlich die sich wandelnden Einflüsse in der Zeit des Umbruchs im Schwedischen widerspiegelt, ein äußerst wichtiges Ergebnis von Inabas Untersuchung. Damit sind sowohl die Erforschung der Besitzverhältnisse im Ostseefinnischen als auch die Genitivund Dativforschung nun vom hinderlichen Druck der Hypothesen über die Entstehung des Genitivs und auch von dem Glauben an den fossilen Charakter des finnischen Dativgenitivs befreit.

Ein zweites wichtiges Ergebnis ist die Nähe des Genitivs beim Ausdruck der Besitzverhältnisse im Südsaamischen, Mordwinischen und Mari.

Ernsthaft $\mathrm{zu}$ beachten ist drittens Inabas Feststellung, dass der livische Dativ mehr gemeinsame
Verwendungsbereiche mit dem finnischen Genitiv hat als bisher angenommen. Obwohl der livische Dativ Einflüsse aus dem Lettischen aufweist, wurde der Dativ nicht aus dem Lettischen entlehnt, und da es in alten Zeiten auch Skandinavier in die livischen Gebiete verschlug, ist es angebracht, auch im Livischen nach alten skandinavischen Einflüssen zu suchen.

Da Nobufumi Inaba in seiner Arbeit eine beeindruckende Korpusuntersuchung über die Besitzverhältnisse in fünf finnischugrischen Genitivsprachen, nämlich in den verschiedenen Epochen der alten finnischen Schriftsprache, im Livischen (Kurland- und Salislivisch), im Südsaamischen, im Mordwinischen (sowohl Erza als auch Mokša) und im Mari (sowohl Wiesen- und Ostmari als auch Bergmari) und darüber hinaus über die Besitzverhältnisse und Besitzsatztypen in der Umbruchszeit des Schwedischen, über die verschiedenen Typen des finnischen Dativgenitivs und ihre Verbreitung sowie über den livischen Dativ und seine Verwendung vorlegt und aufgrund seiner Untersuchung ferner auch Vergleiche dieser Sprachen anstellt, sind seine Belege und Beilagen auch für die Erforschung dieser Sprachen von Nutzen.

Tiit-Rein Viitso 